\title{
DIPSI criteria-cost-effective approach in low resource setup in developing countries
}

\author{
Balaji Vijayam¹ ${ }^{1}{\text { Madhuri S Balaji }{ }^{1} \text {, Meghna Seshiah }}^{2}$, Anitha Rani.A ${ }^{1}$ \\ Dr. V Balaji Dr. V Seshiah Diabetes Care and Research Institute, \\ Aminjikarai, Chennai, Tamil Nadu, India. ${ }^{2}$ The Seven Hills School Cincinnati, Ohio, USA.
}

\begin{abstract}
:
In the present scenario the prevalence of Gestation diabetes is rapidly increasing worldwide. Women with diabetes during pregnancy are not different from other women in their concern for their foetuses, perhaps they need to put more effort, as the slight variation from the normal value might have a major impact on fetal well-being. In India alone, GDM affects five million women each year, which is a huge burden for the health care providers to deal with. Henceforth, screening and diagnostic criteria for testing GDM should be convenient, practically possible and cost effective. DIPSI test is introduced in India should be made as a central point of discussion and consideration. The current review focused on the screening and diagnostic criteria of GDM in low resource setting using DIPSI criteria.
\end{abstract}

DOI: 10.29322/IJSRP.11.09.2021.p11705

http://dx.doi.org/10.29322/IJSRP.11.09.2021.p11705

Key words: GDM, DIPSI, Prevalence.

Introduction:

Gestational Diabetes Mellitus (GDM) poses a significant public health challenge. Worldwide, prevalence of GDM is increasing dramatically, which is an area of intense clinical research. There has been an increase in prevalence, varying by ethnic group and diagnosis criteria used. It is more common in Asia. India beings a diabetic capital also experiencing the increasing prevalence of GDM. Indian women are known to have 11times increased risk of developing GDM when compared to Caucasian women. GDM increased the risk of adverse maternal outcomes.

GDM is defined as "any degree of glucose intolerance with onset or first recognised during pregnancy" [1]. Both maternal and fetal outcomes are associated with it [2,3]. Worldwide, 21.3 million pregnancies are associated with hyperglycaemia and 18.4 million of these result in gestational diabetes mellitus [4]. Pregnancy is considered as a diabetogenic state, with a typical characteristic of increased rate of insulin release, which is associated with the decreased insulin sensitivity at cellular levels. There is increasing state of anti insulinogenic hormones in mid pregnancy which resulted in abnormal glucose tolerance [5]. "Carbohydrate intolerance of variable severity with the onset or first recognition during the present pregnancy" [6]. The definition applies irrespective of whether or not insulin is used for the treatment or the condition persists after pregnancy. Due to the increasing prevalence of GDM, for all pregnant women in India screening is crucial.

Over the period of time, the diagnostic criteria for DM outside pregnancy was evolve and was widely accepted by diabetic organizations worldwide, Perhaps the screening and diagnosis of GDM still remains debatable issue. Nevertheless the decades of dedicated research and a number of international workshops on GDM, there exist a disagreement among the international bodies in view of global approach towards the screening and diagnostic procedures of GDM [7]. In point of fact the guideline for screening and diagnosis for GDM varies among countries and major societies worldwide. Frequently, within countries also there exist the 
lack of agreement between the diabetes societies and obstetric societies, where each recommends different approach [8]. Thus the need of the hour is a robust well validated approach in screening and diagnosing and management of GDM.

The NIH consensus [9] and ADA position statement [10] highlights that bringing uniform approach to the diagnosis of GDM would be highly beneficial to patients, caregivers, and policymakers, which remains a strong consensus. Different screening and diagnostic criteria of GDM, identifies different sets of women with GDM. The guideline would be based on the many factors such as cost concern, infrastructure availability and convenience to patients. The difference of opinion in the guidelines used in various settings might be due to the paucity of information and resource availability

\section{GDM screening \& diagnosis in Low resource settings:}

According to International Diabetes federation (IDF, 2017) majority of Hyperglycaemic incidence was occurred in Low and Middle income Countries (LMICs), associated with limited maternal care resources [11]. In LMIC with limited resource, in spite of the scientific validity of GDM guidelines there exist a limitation in following the same. GDM is a major public health related problem which exist in all levels of economic development, perhaps the instant and pragmatic approach, might limits the resource [12]. In low resource settings, there are many barriers in screening GDM which include both patient related and heath care systems (table 1). In the remote rural areas pregnant women have to travel a longer distance for their routine check- up, thus they doesn't turn up for their regular follow ups. Further screening in fasting state is highly not feasible. In remote areas there is lack of standardized lab facilities and trained phlebotomist to perform the test, as required by the guidelines, thus it pose a challenge in universal screening.

Table 1: Road blocks for screening Gestational Diabetes in low resource settings.

\begin{tabular}{|l|l|}
\hline \multicolumn{1}{|c|}{ Health care system } & \multicolumn{1}{c|}{ Related to Patients } \\
\hline Fragmentation of health services & Low perception on risk of Diabetes \\
\hline Lack of trained phlebotomists & $\begin{array}{l}\text { Contact health care system at the later stage and / or Lack of } \\
\text { follow up visits }\end{array}$ \\
\hline Access to care & $\begin{array}{l}\text { Lack of awareness on GDM and its complications for both mother } \\
\text { and fetus }\end{array}$ \\
\hline Lack of trained health care professionals & Undergoing the OGTT in the fasting state \\
\hline $\begin{array}{l}\text { Lack of diagnostic facilities and standardized } \\
\text { laboratories, Storage and transport of blood samples }\end{array}$ & $\begin{array}{l}\text { Distance of health care facility/ Longer distance to the primary } \\
\text { health care centres and unavailable doctor at a community clinic }\end{array}$ \\
\hline $\begin{array}{l}\text { Lack of standard approach to postpartum } \\
\text { Coming for check up in the fasting state }\end{array}$ \\
\hline $\begin{array}{l}\text { Follow up care OGTT not routinely available } \\
\text { Foetal centric approach to counseling during } \\
\text { pregnancy }\end{array}$ & Socioeconomic circumstances and Income \\
\hline Cost of healthcare services and treatment & Migration between Provinces. \\
\hline $\begin{array}{l}\text { Lack of or poor referral system / feedback } \\
\text { mechanism and follow - up systems }\end{array}$ & $\begin{array}{l}\text { Perception of female body size and weight gain/ loss relation to } \\
\text { pregnancy }\end{array}$ \\
\hline
\end{tabular}

\section{A Single Step Cost effective Procedure:}

DIPSI recommends a 2 hours plasma glucose level greater than or equal to $140 \mathrm{mg} / \mathrm{dl}(7.8 \mathrm{mmol} / \mathrm{dl})$ with oral administration of $75 \mathrm{~g}$ glucose to pregnant women, regardless of the last meal time to identify GDM among pregnant women [13]. GDM diagnosis and treatment is useful in decreasing the maternal complications and improves the health related quality of the life in pregnant women. DIPSI criteria is evidence based, feasible, cost-effective, sustainable and high impact and best for low resource setting. Several international organizations, such as the World Health Organization (WHO), the International Diabetes Federation 
(IDF) and International Federation of Gynecology and Obstetrics (IFGO), have approved DIPSI criteria. According to DIPSI (2009) criteria non-fasting $2 \mathrm{hr}$ venous plasma value of $\geq 140 \mathrm{mg} / \mathrm{dl}$ is considered as a single-step, definitive, screening and diagnostic tool for GDM [13]. In a study conducted among 800 pregnant women with the gestation age of $16-32$ weeks. GDM was diagnosed in $10.9 \%$ in both DIPSI and WHO1999 criteria and reported 100\% sensitivity and specificity of non-fasting DIPSI compared to WHO 1999 criteria in diagnosing GDM. DIPSI guideline is already being followed in South Asian countries [14, 15]. In clinical practise, it is expected to use the simpler and cost effective approaches, which is acceptable by the pregnant women. DIPSI procedure fits in this criteria, where, one time blood sample was drawn after administering $75 \mathrm{~g}$ oral glucose load regardless of the last meal time. Even if the test has to be repeated in the third trimester the cost to perform the test is much less than the cost required for performing the other procedures. The cost per test using DIPSI criteria is approximately 21INR, perhaps it is tripled when using other criteria. Hence it is cost effective and can be used in low resource setups.

\section{Advantage of DIPSI criteria over other methods}

- It is simple one step screening and diagnostic test for GDM and more feasible.

- After oral administration of $75 \mathrm{~g}$ of glucose load, one time blood sample was drawn at $2 \mathrm{hrs}$ to estimate plasma glucose.

- No need to come again in the fasting state.

- If there is a need to repeat the test in each trimester, the cost of DIPSI procedure is less when compared to other recommended procedure.

- $\quad$ Potential to improve compliance.

- $\quad$ Less risk of vomiting after glucose load.

- $\quad$ Cost effective and reliable.

The logic behind this non-fasting state of test is that the glucose levels are affected negligibly by the time of meal in normal glucose tolerant women, perhaps it is not the case in GDM.

In India nearly $70 \%$ of the population lives in rural setup, which has limited basic facility for diagnosing diabetes. In this situation performing OGTT to diagnose GDM is practically not possible. Further the cost involved in performing three blood test is not favored to both health care providers and also to health care seekers. Thus universal screening program for GDM cannot be implemented. In India, the rate of still birth is high, which is one of the cause of GDM, thus there is a need to escalate the diagnosis and the care for GDM as a public health priority (16). There is a requirement for a simple and cost effective test to diagnose GDM. In this situation single time blood sample is cost effective and evidence based as revealed by the pregnancy outcome by Wahi et al [17]. If there is a need to repeat the test in each trimester, the cost of DIPSI procedure is $66 \%$ less when compared to the cost of any other diagnostic procedure. It is a practical in areal time scenario, cost-effective approach, which address the patients' needs [18].

Balaji et al., [19] reported GDM in $14.6 \%$ pregnant women by IADPSG and $13.4 \%$ by DIPSI criteria. According to this study DIPSI is cost-effective without compromising clinical effectiveness. GDM can be diagnosed easily and effectively in any socio-economic setting by the DIPSI criteria, which provides a one step-cost-effective procedure. Dash et al., (2021) compared random $75 \mathrm{~g}$ oral glucose tolerance test (OGTT) by DIPSI criteria with fasting $75 \mathrm{~g}$ OGTT by IADPSG criteria in diagnosing GDM and compare outcomes of patients diagnosed with these two criteria. The study include $24-42$ weeks gestational age of pregnant women, as this is a vulnerable period where the chances of detecting the GDM cases were more when compared to $24-28$ weeks of gestational period $[20,21]$. The study finding showed that incidence of GDM was 12\% with DIPSI and $11.3 \%$ with IADPSG criteria. There is no significant difference in the prevalence rate of GDM, maternal and perinatal outcomes in patients diagnosed with DIPSI and IADPSG method. DIPI is a reliable, feasible and suitable method to diagnose GDM. The DIPSI criteria is just as sensitive as the WHO 2018 criteria in a hospital setting [22]. In a study conducted among 425 pregnant women, GDM was diagnosed in $5.88 \%$, were $1.41 \%, 1.18 \%$ and $3.29 \%$ were diagnosed by DIPSI, WHO 2018 and by both methods respectively. The study This publication is licensed under Creative Commons Attribution CC BY. 
highlighted the sensitivity $(73.68 \%)$ and specificity $(98.5 \%)$ of DIPSI criteria. There exist a good agreement ranged between $60 \%$ to $80 \%$ among DIPSI and WHO 2018 criteria. Hence, the DIPSI criteria is highly comparable with WHO 2018 criteria and can be adopted.

An Indian study found that sensitivity and specificity of DIPSI was $45 \%$ and $87 \%$ whereas for IADPSG it was $40 \%$ and $89 \%$ respectively, the sensitivity of DIPSI was better when compared to IADPSG criteria. The study finding highlighted that in all pregnant women GDM screening GDM is mandatory due its increasing prevalence in India [23]. Similar finding was observed by Geetha et al., where 14\% and 9\% were diagnosed by DIPSI and by IADPSG respectively, whereas $4 \%$ were diagnosed by both criteria. In this study, 5\% of GDM leaves undiagnosed by IADPSG, which can be easily diagnosed by DIPSI [24]. The above mentioned studies were prospective study with small sample size of 144 and 100 respectively. In LMICs where the prevalence of diabetes is high, the universal and early screening with a single step, DIPSI criteria seems to be convenient highly cost effective [25]. In a study conducted by Polur et al., to diagnose GDM, WHO and DIPSI criteria was compared and correlated. The finding showed that out of 149 pregnant women, GDM was diagnosed in 63 cases using WHO and 58 cases using DIPSI, where 52 were diagnosed by both WHO and DIPSI. Thus DIPSI was found to identify 92\% of GDM cases identified by WHO. There exist a significant correlation between DIPSI criteria and WHO $2^{\text {nd }}$ hour, thus DIPSI has all qualities of screening test. Hence it is a simple, cost effective convenient, single, economical, which can be used as both screening and diagnostic evidence based procedure in low recourse countries with high risk population [26].

A study was conducted to validate the single-step, non-fasting 75gm DIPSI GDM criteria in Indian population and was compared with the two-step fasting 100gm glucose challenge through the Carpenter Coustan criteria (CCC). The finding showed there exist a high sensitivity and specificity with negative predictive value and diagnostic accuracy for DIPSI when compared to CCC [27]. In a prospective study conducted in Sri Lanka among 165 women between 24 - 28 weeks of gestation, 20\% and $24.4 \%$ had GDM detected by IADPSG and DIPSI respectively. This study showed low sensitivity for DIPSI when compared to IADPSG criteria, the area under the ROC curve is 0.80 , which indicates its utility for GDM diagnoses. Furthermore DIPSI is a single step procedure which is highly acceptable among women [28]. The prevalence rate of GDM was accessed using IADPSG and DIPSI criteria, the finding doesn't find any significant difference between the prevalence rates of GDM among the two criteria [29].

DIPSI criteria is simple to perform and execution and further the patient friendly is very close to international consensus. India have a diverse and variable population, further studies are required from different centers of India with larger cohort size, is the need of the hour to develop a national consensus to identify GDM in Indian scenario. The effectiveness of GCT for screening and diagnosing GDM irrespective of the last meal time has long been a matter of debate. The major advantage of DIPSI criteria is that the pregnant women need not be in fasting state, thus they won't get morning sickness or vomiting while administering oral glucose, no waiting period and cause minimal disturbance to their routine activities. DIPSI can be used for both screening and diagnostic purposes. In case if DIPSI procedure to be repeated, the cost to perform the test is comparatively less than other diagnostic criteria and also requires less preparation.

\section{Conclusion}

India's Contributions have made an impact on GDM diagnosis and management. The risk of GDM is higher among Indian women, when compared to other ethnics. Thus Universal screening is necessary in the Indian context. Customized Screening might be necessary in certain situation. The practical applicability of the screening and diagnosis of GDM should be based on the availability and cost effectives with the existing health care facility. Eventually it should be par with the internationally accepted criteria. Taken all the above mentioned considerations DIPSI is most suitable in detecting and screening GDM in low resource settings. DIPSI criteria requires one time estimation of plasma glucose to diagnose GDM. Thus it is cost effective procedure and can be recommended to all pregnant women from all socioeconomic status. In community, $75 \mathrm{~g}$ GCT irrespective of the last meal 
International Journal of Scientific and Research Publications, Volume 11, Issue 9, September 2021

ISSN 2250-3153

timing is recommended, GDM diagnosed if $2 \mathrm{hr} P G \geq 140 \mathrm{mg} / \mathrm{dl}$ and Single test that is cost-effective and reliable. In clinics fasting and $2 \mathrm{hr}$ plasma glucose with $75 \mathrm{~g}$ OGTT may be performed. Screening for glucose intolerance at the earliest opportunity during pregnancy is mandatory. Repeat Screening when negative. A multi-disciplinary approach is necessary in managing women with GDM. Future research can be focused on the evidence based procedures to address the existing research gap in the field of GDM.

\section{References:}

1. World Health Organization. Diagnostic criteria and classification of hyperglycaemia first detected in pregnancy. No. WHO/NMH/MND/13.2. World Health Organization, 2013.

2. Jovanovic-Peterson, Lois, and Charles M. Peterson. "Pregnancy in the diabetic woman: guidelines for a successful outcome." Endocrinology and metabolism clinics of North America 21.2 (1992): 433-456.

3. Hod, Moshe, et al. "The International Federation of Gynecology and Obstetrics (FIGO) Initiative on gestational diabetes mellitus: A pragmatic guide for diagnosis, management, and care\#." International Journal of Gynecology \& Obstetrics 131 (2015): S173-S211.

4. International Diabetes Federation. The IDF approach for care and management of gestational diabetes: wings Profet summary report. Malanda B, editor, 2017.

5. Danilenko-Dixon, Diana R., et al. "Universal versus selective gestational diabetes screening: application of 1997 American Diabetes Association recommendations." American journal of obstetrics and gynecology 181.4 (1999): 798-802.

6. Geneva, Switzerland. "Definition, diagnosis and classification of diabetes mellitus and its complications: report of a WHO Consultation. Part 1: Diagnosis and classification of diabetes mellitus." World Health Organisation (1999).

7. Holt, R. I., M. A. Coleman, and D. R. McCance. "The implications of the new International Association of Diabetes and Pregnancy Study Groups (IADPSG) diagnostic criteria for gestational diabetes." Diabetic medicine: a journal of the British Diabetic Association 28.4 (2011): 382-385

8. Leary, Joyce, David J. Pettitt, and Lois Jovanovič. "Gestational diabetes guidelines in a HAPO world." Best practice \& research Clinical endocrinology \& metabolism 24.4 (2010): 673-685.

9. Vandorsten, James P., et al. "NIH consensus development conference: diagnosing gestational diabetes mellitus." NIH consensus and state-of-the-science statements 29.1 (2013): 1-31

10. American Diabetes Association. "Diagnosis and classification of diabetes mellitus." Diabetes care 37.Supplement 1 (2014): S81-S90.

11. Cho, NH1, et al. "IDF Diabetes Atlas: Global estimates of diabetes prevalence for 2017 and projections for 2045." Diabetes research and clinical practice 138 (2018): 271-281.

12. Metzger, B. E., et al. "International Association of Diabetes \& Pregnancy Study Groups (IADPSG) Consensus Panel Writing Group and the Hyperglycemia \& Adverse Pregnancy Outcome (HAPO) Study Steering Committee. The diagnosis of gestational diabetes mellitus: new paradigms or status quo." J Matern Fetal Neonatal Med 25.12 (2012): 2564-2569.

13. Anjalakshi, C., et al. "A single test procedure to diagnose gestational diabetes mellitus." Acta diabetologica 46.1 (2009): $51-54$

14. Dias, Thiran, et al. "Comparing different diagnostic guidelines for gestational diabetes mellitus in relation to birthweight in Sri Lankan women." Frontiers in endocrinology 9 (2018): 682.

15. Riaz, Musarrat, et al. "Frequency of gestational diabetes mellitus using DIPSI criteria, a study from Pakistan." Clinical Epidemiology and Global Health 7.2 (2019): 218-221.

16. Pattinson, Robert, et al. "Stillbirths: how can health systems deliver for mothers and babies?." The Lancet 377.9777 (2011): $1610-1623$ 
17. Wahi, Preeti, et al. "Prevalence of gestational diabetes mellitus (GDM) and its outcomes in Jammu region." $J$ Assoc Physicians India 59.4 (2011): 227-30.

18. Yogev, Yariv, Boyd E. Metzger, and Moshe Hod. "Establishing diagnosis of gestational diabetes mellitus: Impact of the hyperglycemia and adverse pregnancy outcome study." Seminars in Fetal and Neonatal Medicine. Vol. 14. No. 2. WB Saunders, 2009.

19. Balaji, V., et al. "Diagnosis of gestational diabetes mellitus in Asian-Indian women." Indian journal of endocrinology and metabolism 15.3 (2011): 187.

20. Wahi, Preeti, et al. "Prevalence of gestational diabetes mellitus (GDM) and its outcomes in Jammu region." J Assoc Physicians India 59.4 (2011): 227-30.

21. Puttaraju, Chandana M., and Manorama Eti. "WHO 75 gram OGTT-A single step procedure for screening and diagnosis of gestational diabetes mellitus." International Journal of Reproduction, Contraception, Obstetrics and Gynecology 4.6 (2015): $2022-2027$.

22. Shrestha, Biloni, and L. Pokhrel. "Comparative study of DIPSI and WHO 2018 criteria for diagnosis of GDM." Nepal Medical College Journal 22.1-2 (2020): 13-17.

23. Srinivasan, Swathy, and Rani P. Reddi. "Comparative study of DIPSI and IADPSG criteria for diagnosis of GDM." International Journal of Reproduction, Contraception, Obstetrics and Gynecology 7.3 (2018): 933.

24. Geetha, N., and K. G. Sangeetha. "Comparison of IADPSG and DIPSI criteria for diagnosis of gestational diabetes mellitus." IOSR Journal of Dental and Medical Sciences 15 (2016): 1-4.

25. Saxena, Pikee, Sakshi Miglani, and Aruna Nigam. "Screening and Diagnosis of Gestational Diabetes Mellitus: from Controversy to Consensus." Current Research in Diabetes \& Obesity Journal 2.5 (2017): 99-102.

26. Polur, Havilah, et al. "Diabetes in pregnancy Study group in India (DIPSI)-a novel criterion to diagnose GDM." International Journal of Biochemistry Research \& Review (2016): 1-6.

27. Khan, Shazia, et al. "Evaluation of the diabetes in pregnancy study group of India criteria and Carpenter-Coustan criteria in the diagnosis of gestational diabetes mellitus." Turkish journal of obstetrics and gynecology 15.2 (2018): 75.

28. GRMUGP, Jayawardena, et al. "Effectiveness of Diabetes in Pregnancy Study Group of India (DIPSI) criteria in detecting Gestational Diabetes among women attending a Tertiary Care Hospital in Sri Lanka." International Journal of Obstetrics and Gynecology 9.1 (2021): 1-7.

29. DasMukhopadhyay, Lipika, Sudhindra Mohan Bhattacharya, and Ankita Dey. "Prevalence of gestational diabetes mellitus utilizing two definitions." The Journal of Obstetrics and Gynecology of India 70.3 (2020): 245-247. 